\title{
A MOBILE APPLICATION FOR SUGARCANE PLANT HEALTH MONITORING
}

\author{
Sapna \\ Dept. of Computer Science and Engineering \\ Centre for Post Graduate Studies \\ V.T.U, Belagavi
}

\begin{abstract}
Recognition of plant diseases early is one of the solution for preventing the losses in the yield and measure of agricultural product. The study of plant diseases signify the visually visible patterns seen on the plant. Health monitoring and disease detection on plant is very critical for sustainable agriculture. It is not easy to monitor the plant diseases manually. It needs lot of work, and expertise in the plant diseases, and in addition require the extreme processing time. Therefore, image processing is used for the identification of plant diseases. Disease detection involves the steps like image acquisition, image preprocessing, image segmentation, feature extraction and classification. Client Mobile application which capture image and send it to server and server executes the task and send back the result regarding the disease, symptoms and remedies on client mobile application. The technique used for the detection of plant diseases is to analyze plant leaves. It furthermore discusses about some segmentation and feature extraction algorithm used in the plant disease detection.
\end{abstract}

Keywords-Image acquisition, Segmentation, Feature extraction, Probabilistic Neural Network, Android application

\section{INTRODUCTION}

India is an agriculture nation where in about $72 \%$ of the people depend on agriculture. Farmers contain huge range of diversity intended for choosing different appropriate crop and finding the proper pesticides used for plant. Disease on plant leads toward reduction in both the quality and quantity of agricultural yield. The study of plant disease refers to the study of visually apparent pattern on the plants. Monitoring of health and disease on plant plays vital role in successful development of crops in the farm. In early days, an analysis and the monitoring of plant diseases were made by hand through the skilled person in that field. This requires great amount of work and in addition it requires experiment time. The image processing techniques can be used in the plant disease detection. the majority of the disease symptoms are seen on the leaves, stem and fruit. The plant leaf for the detection of disease is measured which shows the disease symptoms. This paper describes an image processing technique used for plant disease detection.

The system consists of a mobile application, which will allow the farmers to take images of plants by their mobile phones and send it to a central server where the central system in the server will analyze the pictures

\author{
Dr. S A Angadi \\ Professor in Dept. of Computer Science and Engineering \\ Director (I/C) ITISMU \\ V.T.U, Belagavi
}

based on visual symptoms using image processing algorithms in order to measure the disease type. The specialist group will be obtainable to verify the status of report and their knowledge, which will be sent to the farmer as a notification in the application. The purpose of this project is to monitor diseases on plant leaves and recommend better solution for healthy yield and productivity. The diseases are caused through bacteria and virus and it can take place in both nursery and main field stage. The diseases include Red rot, Eye spot, Ring spot, wilt, Grassy shoot, Smut Leaf scald disease, Red striped disease, Mosaic disease, Pokkahboeng, Rust, Sugarcane yellow leaf disease etc.

The Mobile Application that can help to bring timely and made advice to farmers. An android mobile app that uses to identify plant diseases when the user uploads an image of damage leaf through their smart phone. It provides advisory on disease management including biological and chemical control measures and symptoms. The app also will provide the latest management advice for all major diseases. The app employs in real time so the farmer can be an active participant in disease diagnosis and crop health management, leading to more yields for smallholder farmers.Section I includes the Introduction. Section II focuses on the Literature Survey. Section III highlights the Proposed Methodology while Section IV presents the Experimentation and Results and Section V concludes the paper.

\section{RELATED WORK}

The literature has many reported walks in the domain. [1] H. Al-Hiary et al. In this paper, respectively, the applications of K-means clustering and Neural Networks (NNs) have been formulated for clustering and classification of diseases that affect on plant leaves. Recognizing the disease is mainly the purpose of the proposed approach. Thus, the proposed Algorithm was tested on five diseases which influence on the plants; they are: Early scorch, Cottony mold, ashen mold, late scorch, tiny whiteness.

[2],P.Revathi, M.Hemalatha. In this paper, addressed how the disease analysis is possible for the cotton leaf diseases detection, the analysis of the various diseases present on the cotton leaves can be effectively detected in the early stage before it will damage the whole plant. 


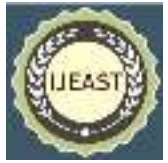

Here the technique presented can able to detect the disease more accurately, The Viderbha and Marathwada Region of Maharashtra state is main producer of cotton, where if this model is applied, we can say that, we can archive good productivity by preventing the various diseases present on the leaves of cotton plant.

[3] Pooja.D.Gulshetty et al. have worked on Plant Disease Detection via Image Processing recognition of the plant diseases is the key to preventing the losses in the yield and measure of agricultural product. The analysis of the plant diseases signify the visually noticeable patterns seen on top of the plant. Health monitoring and disease recognition on plant is very significant for sustainable agriculture. It is very difficult to monitor the plant diseases manually. It requires tremendous amount of work, and expertize in the plant diseases, and also require the excessive processing time. Hence, image processing is used for the detection of plant diseases. Disease detection involves the steps like image acquisition, image pre-processing, image segmentation, feature extraction and classification. The methods used for the detection of plant diseases using their leaves images. It also discusses some segmentation and feature extraction algorithm used in the plant disease detection.

[4] S. Arivazhagan et al. have worked on Detection of unhealthy region of plant leaves and classification of plant leaf diseases using texture features. Plant diseases have turned into a dilemma as it can cause important reduction in both quality and quantity of agricultural products. Automatic detection of plant diseases is an essential research topic as it may prove benefits in monitoring large fields of crops, and thus automatically detect the symptoms of diseases as soon as they appear on plant leaves. The proposed system is a software solution for automatic detection and classification of plant leaf diseases. The developed processing scheme consists of four main steps, first a color transformation structure for the input RGB image is created, then the green pixels are masked and removed using specific threshold value followed by segmentation process, the texture statistics are computed for the useful segments, finally the extracted features are passed through the classifier. The algorithm's efficiency can successfully detect and classify the examined diseases with an accuracy of $94 \%$. Experimental results on a database of about 500 plant leaves confirm the robustness of the proposed approach.

The above state of the art indicates that the disease detection is a problem that needs technological solution. The problem attempted in this work is described as follows.

Problem statement : To design a sugarcane leaf disease recognition system and to determine its disease using its color and texture features. System consists of two phases Training and Testing. Training phase includes: Data
Acquisition, Image Pre-processing, Segmentation, Feature extraction Testing phase includes: Leaf Classification, leaf disease Detection. Mobile Application for input and displaying output

\section{PROPOSED METHOD}

There are two phases one is client side processing as shown (Fig. 1)) and another is server side processing as shown (Fig. 2)).

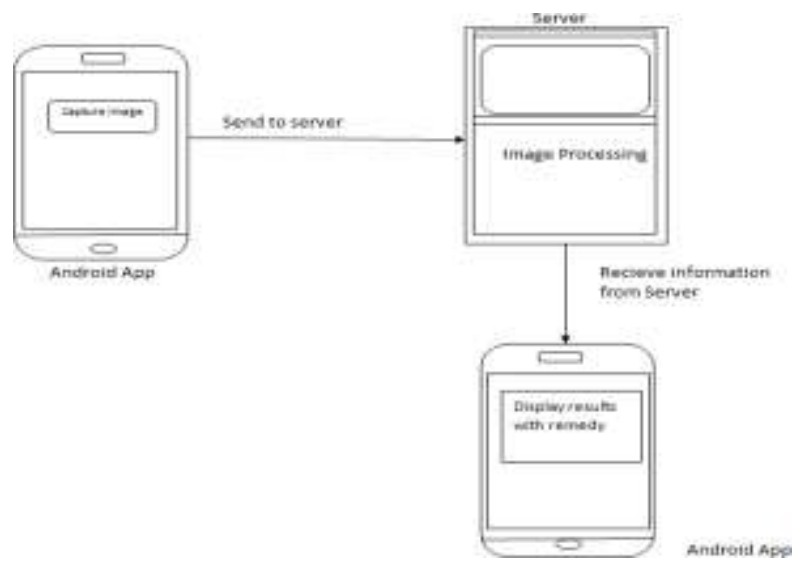

Fig 1): Client side process

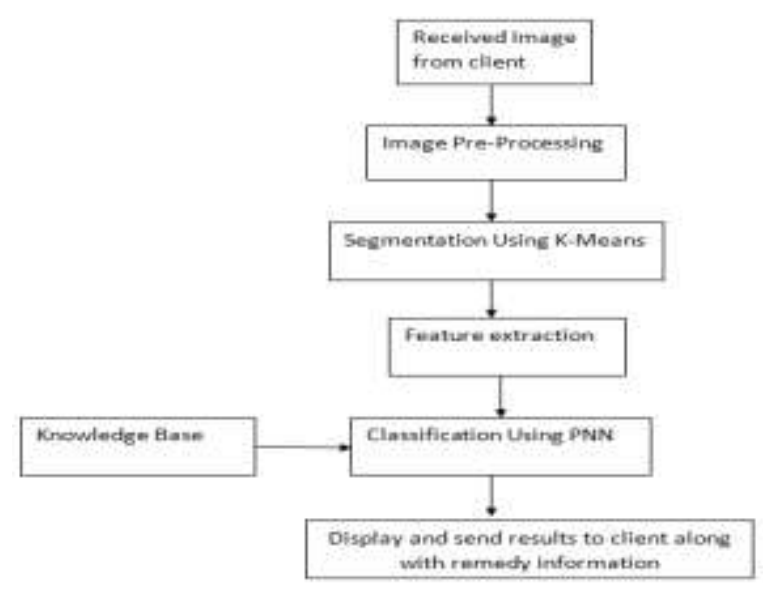

Fig 2): Server side process which includes basic steps for plant disease detection and classification.

The client side process includes Android Mobile application where the user will capture the diseased leaf or query leaf which will be send to the server by clicking send button. The server will process the request and execute the given task and send back the result to an same client application. The result will be in the form of name of the leaf diseased which was captured by the mobile user and also displays remedies of respective diseases.

\section{A] Image Acquisition}

The images of the plant leaf are captured through the camera. This image is in RGB (Red, 


\section{International Journal of Engineering Applied Sciences and Technology, 2019 \\ Vol. 4, Issue 3, ISSN No. 2455-2143, Pages 289-293 \\ Published Online July 2019 in IJEAST (http://www.ijeast.com)}

Green and Blue) form. Color transformation structure for the RGB leaf image is created, and a device-independent color space transformation for the color transformation structure is applied. Image Acquisition is the initial condition for the work flow series of image processing because as processing is possible only with the help of an image. The image obtained is entirely natural and is the consequence of any hardware which was handled to produce it. It takes the image as input for further processing.

\section{B] Image Pre-processing}

To remove noise in image or other object

removal, different pre-processing technique is considered. Image clipping i.e. cropping of the leaf image to get the interested image region. Image smoothing is done using the smoothing filter. Image enhancement is carried out for increasing the contrast, the RGB images into the grey images using color conversion.

\section{C] Image Segmentation}

Segmentation means partitioning of image into various part of same features or having some similarity. The segmentation can be done using various methods like otsu' method, k-means clustering, converting RGB image into HIS model etc. but here $\mathrm{K}$ means clustering is used.

K-means clustering: The K-means clustering is used for classification of object based on a set of features into $\mathrm{K}$ number of classes. The algorithm for $\mathrm{K}$-means Clustering:

Basic Euclidean distance metric Let $\mathrm{X}=$ $\{\mathrm{x} 1, \mathrm{x} 2, \mathrm{x} 3, \quad, \mathrm{xn}\}$ be the set of data points and $\mathrm{V}=$ $\{\mathrm{v} 1, \mathrm{v} 2, \mathrm{vc}\}$ be the set of centers.

1. Select 'c' cluster centers randomly.

2. Calculate the distance between each data point and cluster centers using the Euclidean distance metric as follows

$$
\text { Dist }_{X Y}=\sqrt{\sum_{k=1}^{m}\left(X_{i k}-X_{j k}\right)^{2}}
$$

3. Data point is assigned to the cluster center whose distance from the cluster center is minimum of all the cluster centers.

4. New cluster center is calculated using:

$$
V_{\mathrm{i}}=\left(\frac{1}{C i}\right) \sum_{1}^{\mathrm{Cl}} x_{\mathrm{i}}
$$

where, 'ci' denotes the number of data points in ith cluster.

5. The distance between each data point and new obtained cluster centers is recalculated.
6. If no data point was reassigned then stop, otherwise repeat steps from 3 to 5 .

\section{D] Feature Extraction}

Feature extraction plays an important role for identification of an object. In many application of image processing feature extraction is used. Color, texture, morphology, edges etc. are the features which can be used in plant disease detection. Here color and textural feature extraction is done.

Color feature : Mean, Standard deviation, Variance of RGB(RedGreenBlue), CMY(CyanMegentaYellow) values Texture feature : LBP values, Hu moments.

Local Binary Pattern (LBP) is a straightforward yet very efficient texture operator which labels the pixels of an image by thresholding the neighborhood of each pixel and considers the outcome as a binary number. Due to its discriminative control and computational ease, LBP texture operator has become a popular approach in various applications. It can be seen as a unifying approach to the traditionally divergent statistical and structural models of texture analysis. possibly the most important property of the LBP operator in real-world applications is its robustness to monotonic gray-scale changes caused, for example, by illumination variations. Another important property is its computational ease, which makes it possible to analyze images in challenging real-time settings.

Hu Moments: Image moments capture information about the form of a blob in a binary image since they contain information on the intensity $\mathrm{I}(\mathrm{x}, \mathrm{y})$, as well as position $\mathrm{x}$ and $y$ of the pixels. Central moments are translations invariants and normalized central moments are both translation and scale invariant. $\mathrm{Hu}$ moments(or rather hu momemt invariants) are set of 7 numbers calculated using central moments so as invariant to image transformation. The first 6 moments proved to be invariant to translation, scale and rotation and reflection. where the $7^{\text {th }}$ moment's sign changes for image reflection.

\section{F ] Classification:}

Classification is very active research and application field of neural networks. Neural Networks are considered as robust classifier. Classification is an important feature to separate huge datasets into classes intended for Rule generation, Decision Making, Pattern recognition, Dimensionality Reduction, Data Mining etc. The Neural networks have emerged as an key tool for classification. There are various classification algorithms available although here the classification algorithm used is Probabilistic Neural Network(PNN). Figure(3) shows an architecture of PNN [11]. 
International Journal of Engineering Applied Sciences and Technology, 2019

Vol. 4, Issue 3, ISSN No. 2455-2143, Pages 289-293

Published Online July 2019 in IJEAST (http://www.ijeast.com)

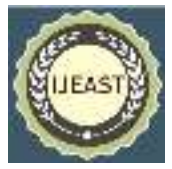

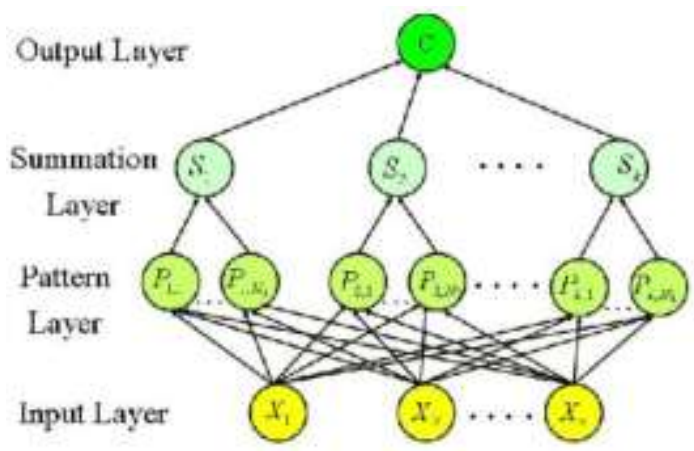

Fig 3): PNN Architecture

A probabilistic neural network (PNN) is predominantly a classifier it map any input pattern to a number of classifications and it can be forced into a more general function approximate. A PNN is an implementation of a statistical algorithm called kernel discriminate analysis in which the operations are organized into a multilayered feed forward network with four layers.

- Input Layer: It transfers input to the hidden layer. (Extracted Features from the dataset are supplied at this point).

- Hidden Layer: There are overall $n$ Neurons in Hidden Layer.

They are grouped based on their equivalent class. If there are $\mathrm{C}$ Classes and $\mathrm{n}$ Neurons then in every group there will be $\mathrm{n} / \mathrm{C}$ neurons.

Output $\mathrm{X}$ at each neuron will be computed by a probability density function ( generally used Gaussian distribution).

Hence $(x)=1 / 2 \sigma \sqrt{ } 2 \prod \exp \{-((\|\mathrm{x}-x j\|) 2 / 2 \sigma 2)\}$

Where $\mathrm{i}=1$........... $\mathrm{n}$ (Neurons in Hidden Layer)

$\mathrm{j}=1$.............. $\mathrm{k}$ (Number of Inputs in Input Layer)

$\sigma$ is the smoothing parameter (values depends on the data $\sigma$ set or estimated heuristically)

- Pattern Layer/Summation Layer: every neurons which belongs to that class will be summing up at this point.

fi $x=(x) l j=1$

where $\mathrm{i}=1$ C (Classes)

1 is the number of neurons which belongs to that class.

- Output Layer: It decides in which class test sample belongs by comparing the $\mathrm{f}$ values of the pattern layer.

If $\boldsymbol{f} \boldsymbol{i} \boldsymbol{x} \geq \boldsymbol{f} \boldsymbol{j} \boldsymbol{x} / /$ Given $\mathrm{i} \neq \mathrm{j}$

Then $\mathrm{x} \in \mathrm{i}$ (eth number of class)

Else

$\mathrm{x} \in \mathrm{j}$ (eth number of class)

End

\section{EXPERIMENTAL RESULTS}

The complete system is designed using matlab and Android operating system . A diseased leaf samples are capture by different mobile devices at different resolutions. The Figure (4) represents Loading module, loading of image is done through online or real time. The user can load the image through storage or real time using the android phone application. The Figure (5) represents the output with disease name and prevention measures is appeared on to the android mobile phone.
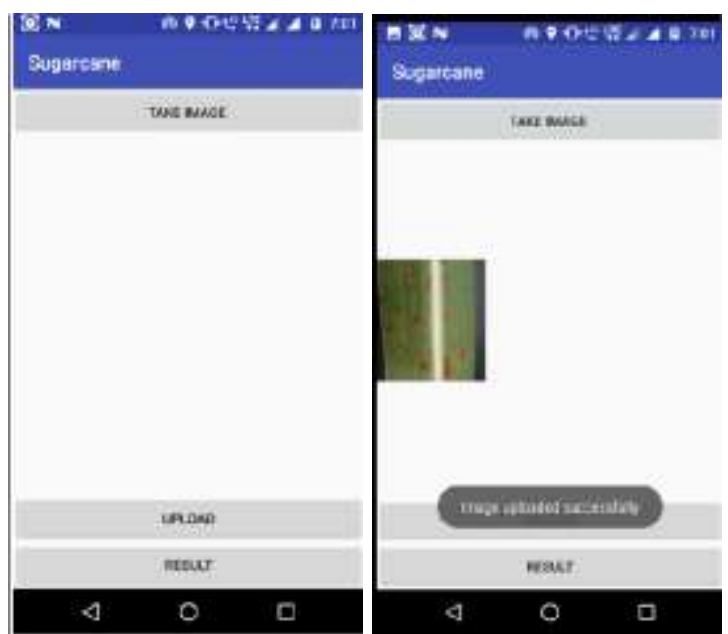

Fig 4): Front end of Android App For testing an image and take leaf image for testing and upload it on server

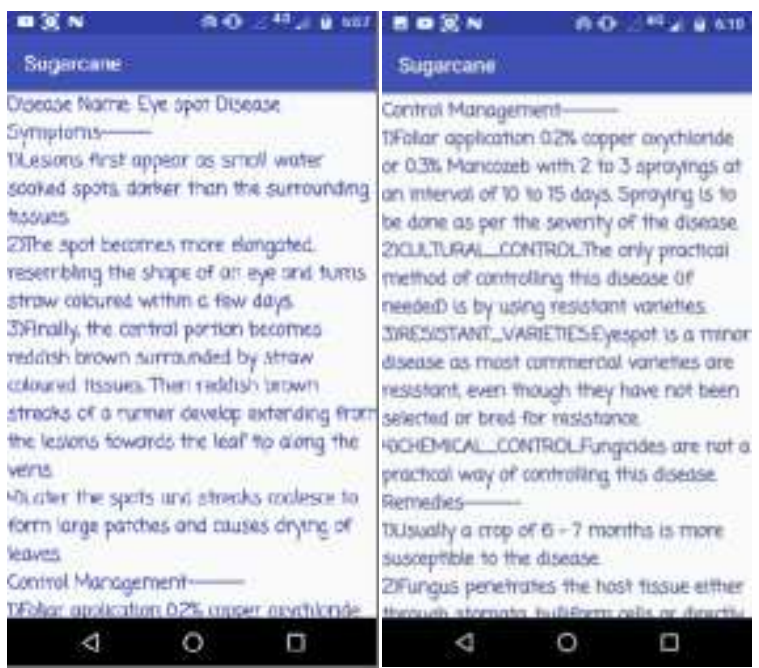

Fig 5): Result is displayed on user Mobile Application with disease, Symptoms, Control management and Remedies

The proposed system uses PNN for identifying sugarcane plant leaf disease. I have used 75 sample images for training PNN network and experiments were conducted on 26 images. 
International Journal of Engineering Applied Sciences and Technology, 2019

Vol. 4, Issue 3, ISSN No. 2455-2143, Pages 289-293

Published Online July 2019 in IJEAST (http://www.ijeast.com)

\begin{tabular}{|c|c|c|c|c|}
\hline Disease name & $\begin{array}{l}\text { Total No. of } \\
\text { testing Images }\end{array}$ & Success & Failed & Aceuracy \\
\hline Red rot disease & 6 & 4 & 2 & $85 \%$ \\
\hline EyeSpot disease & 5 & 4 & 1 & $89 \%$ \\
\hline Rust discase & 6 & 6 & 0 & $90 \%$ \\
\hline Yellow leaf disease & 5 & 3 & 2 & $83 \%$ \\
\hline Healthy leaves & 5 & 4 & 1 & $92 \%$ \\
\hline
\end{tabular}

Table 1): Testing result analysis

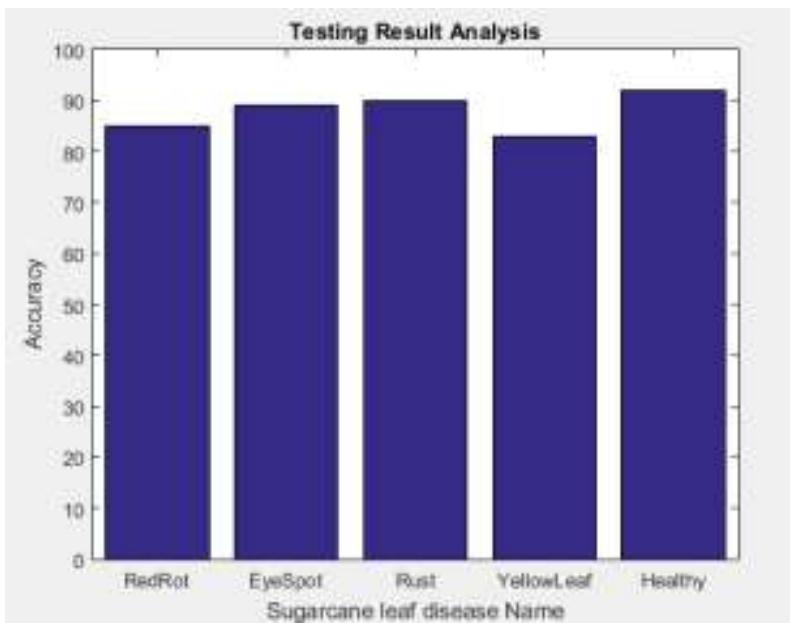

Fig.6) Bar graph on testing results

\section{CONCLUSION}

The proposed system is a detection and classification of the sugarcane plant leaf disease using image processing and android mobile application. Farmer can take the leaf image by using camera to observe the leaf status whether leaf has any disease symptoms or healthy. This will make farmer to take decision before any disease occur to plant leaf. An uploaded image is segmented using $\mathrm{K}$ means clustering technique and color and texture features are extracted. We have trained the neural network by using PNN. The PNN classifier will identify the disease and server will display the result on android mobile application with symptoms, control management and remedies which will help farmers to healthy yield and productivity. We have worked on four diseases redrot, eyespot, rust, yellow leaf and healthy leaves the result accuracy is $88 \%$.

\section{ACKNOWLEDGMENT}

I would like to express my special thanks of gratitude to my guide and HOD Dr S.A.Angadi, who gave me the opportunity to do this wonderful project on the topic A Mobile Application For Sugarcane Plant Health Monitoring which also helped me in doing lots of research and I came to know many things I am really thankful to them and even my friends.

\section{REFERENCES}

[1] H. Al-Hiary, S. Bani-Ahmad, M. Reyalat, M. Braik and Z. ALRahamneh,2012, "Fast and Accurate Detection and Classification of Diseases".

[2] P.Revathi M.Hemalatha, 2012, "Classification of Cotton Leaf Spot Diseases Using Image Processing Edge Detection Techniques".

[3] Manisha A. Bhange, Prof. H. A. Hingoliwala, , 2015 “A Review of Image Processing for Pomegranate Disease Detection", Vol. 6.

[4] Santanu Phadikar and Jaya Sil, , 2008. "Rice Disease Identification using Pattern Recognition Techniques"

[5] D.N.D.Harini, D.Lalitha Bhaskari, 2011. "Identification of Leaf Diseases in TomatoPlant Based on Wavelets and PCA"

[6] Paulraj M P,C R Hema",2009" Color Recognition Algorithm using a Neural Network Model in Determining the Ripeness of a Banana.

[7] Prathamesh Avalekar, L. S. Admuthe , July 2018 "Tomato grading system based on colour models by using neural network", Volume: 05 Issue: 07.

[8] Shweta Joshi, Gayatri Jamadar, Sagar Nachan, R.R.Itkarkar, 2015 "Chilli Disease Detection”, Volume- 3, Issue-2.

[9] Arti N. Rathod, Bhavesh A. Tanawala, Vatsal H. Shah,-2014"Leaf Disease Detection Using Image Processing And Neural Network".

[10] S. Arivazhagan, R. Newlin Shebiah, S. Ananthi, S. Vishnu Varthini, - 2013"Detection of Unhealthy region of Plant Leaves and Classification of Plant Leaf Diseases using Texture Features".

98747007753a9bb6d05f752e.pdf 